\title{
Valence band structure of AIN probed by photoluminescence
}

\author{
A. Sedhain, J. Y. Lin, and H. X. Jiang ${ }^{\text {a) }}$ \\ Department of Physics, Kansas State University, Manhattan, Kansas 66502-2601, USA
}

(Received 19 November 2007; accepted 14 January 2008; published online 31 January 2008)

\begin{abstract}
Deep ultraviolet photoluminescence (PL) was employed to probe the valence band structure of AlN epilayers grown by metal organic chemical vapor deposition on $c$-plane sapphire substrates. At $10 \mathrm{~K}$, in addition to the dominant emission peak at $6.050 \mathrm{eV}$ polarized in the $\mathbf{E} \| \mathbf{c}$ direction, we observed two additional emission peaks at 6.249 and $6.262 \mathrm{eV}$ polarized in the $\mathbf{E} \perp \mathbf{c}$ direction. These two emission lines are assigned to the recombination of free excitons related to the $B$ and $C$ valence bands. A more comprehensive picture of the valence band structure of AlN is thus directly obtained from the PL measurements. (C) 2008 American Institute of Physics.
\end{abstract}

[DOI: $10.1063 / 1.2840176$ ]

AlN has the widest direct band gap $(\sim 6.1 \mathrm{eV})$ among the nitride semiconductors and offers a continuous band-gap variation through the alloying and heterostructure design with $\mathrm{GaN}$ and $\mathrm{InN}$. It is an ideal material for the development of UV-light sources (detectors) operating in the wavelength range down to $200 \mathrm{~nm}$. The electrical and optical properties of semiconductors are mainly determined by the electronic band structure near the $\Gamma$ point. Information regarding the band structure of AlN is fundamentally important for the understanding of the basic properties of AlN and its device applications. Due to an increasing interest in AlN in recent years, several theoretical ${ }^{1-3}$ and experimental ${ }^{4-6}$ studies on the fundamental optical properties of AlN have been reported.

The valence band of wurtzite (WZ) III-nitrides with $\Gamma_{15}$ symmetry splits into three levels $(A, B$, and $C$ valence bands in the order of increasing emission energies). Hexagonal symmetry of the WZ structure induces crystal-field splitting into twofold degenerate $\Gamma_{6}$ state and nondegenerate $\Gamma_{1}$ state. ${ }^{1,7}$ Spin-orbit interaction further splits $\Gamma_{6}$ state into $\Gamma_{9}$ and $\Gamma_{7}$ states. Magnitude and nature of crystal-field splitting parameter $\left(\Delta_{\mathrm{CR}}\right)$ in WZ AlN depend on deviations of its lattice constants' ratio $c / a$ and internal $u$ parameter from their values for an ideal WZ structure. ${ }^{2} \mathrm{AIN}$ has a large negative $\Delta_{\mathrm{CR}},{ }^{7,8}$ so that the energy level of $\Gamma_{1}$ is higher than that of $\Gamma_{6}$. By examining $\mathrm{GaN}$ and all other semiconductors with $\mathrm{WZ}$ structure, we notice that $\Delta_{\mathrm{CR}}$ is generally small and positive so that the energy level of $\Gamma_{6}$ is always higher than that of $\Gamma_{1}$. ${ }^{9}$ This unusual ordering of the valance bands gives rise to unique optical properties of AlN. The recombination of excitons associated with the $A, B$, or $C$ valence bands has different probabilities for different polarizations of absorbed or emitted light. ${ }^{1}$ The emission spectrum in AlN has a dominant $\Pi$-polarized $(\mathbf{E} \| \mathbf{c})$ emission $^{1,9}$ in contrast to the dominant $\sigma$ polarized $(\mathbf{E} \perp \mathbf{c})$ emissions in GaN. ${ }^{9}$ These optical polarization properties may be exploited for polarization filtering, polarized emission in AlN based light emitting diodes, polarization-sensitive photodetectors, and AlN based TM mode laser in the future.

The $A$ exciton transition in AlN was experimentally observed by photoluminescence (PL) and cathodoluminescence (CL) measurements. ${ }^{4,9}$ There were also several reports from

\footnotetext{
${ }^{a)}$ Electronic mail: jiang@phys.ksu.edu.
}

optical reflectance measurements on the observation of $A, B$, and $C$ excitonic transitions. ${ }^{5-7}$ However, the $B$ and $C$ excitonic transitions have never been directly observed by PL measurement. Recently, by increasing the thickness of the epilayers, we have achieved AlN epilayers with a reduced dislocation density. ${ }^{10}$ The fabricated metal-semiconductormetal photodetectors based on these AIN epilayers exhibited a peak responsivity at $200 \mathrm{~nm}$ with a sharp cutoff wavelength at $207 \mathrm{~nm}$ and extremely low dark currents $(\sim 50 \mathrm{fA}$ at $300 \mathrm{~V}) .^{10,11}$ In this work, we report on the studies of the valence band structure of AlN based on the observation of three well resolved excitonic emission peaks in high quality AlN epilayers directly probed by polarization resolved PL.

AlN epilayers with a thickness of $4 \mu \mathrm{m}$ were grown on $c$-plane sapphire substrates by metal-organic chemical vapor deposition. ${ }^{10}$ Deep UV PL measurement was employed to probe the excitonic transitions and the polarization of emitted light. The excitation light source was a frequency quadrupled Ti-sapphire laser (with more than $90 \%$ vertical polarization), $76 \mathrm{MHz}$ repetition rate, $100 \mathrm{fs}$ pulse width, and photon energy set around $6.29 \mathrm{eV} .^{12}$ The collected emission was dispersed by a $1.3 \mathrm{~m}$ monochromator. The sample was held in a stage with its $c$ axis (c) pointing in the vertical direction perpendicular to the sample surface and the excitation laser was incident at very small angle with respect to the sample surface, as illustrated in the inset of Fig. 1(a). A polarizer was used in front of the slit of the monochromator to resolve $\mathbf{E} \| \mathbf{c}(\Pi)$ or $\mathbf{E} \perp \mathbf{c}(\sigma$ or $\alpha)$ components.

Figure 1 compares the low temperature PL spectra of an AlN epilayer collected for the emitted light with electric field component (E) (a) parallel $(\mathbf{E} \| \mathbf{c})$ and (b) perpendicular $(\mathbf{E} \perp \mathbf{c})$ to the $c$ axis. The intensity of the emission peak at $6.050 \mathrm{eV}$ for the $\mathbf{E} \| \mathbf{c}$ polarization is much stronger than that for the $\mathbf{E} \perp \mathbf{c}$ polarization. The emission lines at 5.95, 5.84, and $5.73 \mathrm{eV}$ in Fig. 1(b) marked as $1 \mathrm{LO}, 2 \mathrm{LO}$, and $3 \mathrm{LO}$ are one, two, and three phonon replicas of the dominant $6.05 \mathrm{eV}$ emission line in AIN with a LO phonon energy of about $110 \mathrm{meV}$. The peak at $6.18 \mathrm{eV}$ is the Raman line due to the excitation laser set at $6.29 \mathrm{eV}$. The dominant emission line at $6.05 \mathrm{eV}$ has a linewidth of about $29 \mathrm{meV}$. Previous theoretical calculation ${ }^{4}$ showed that the $A$ valence band related band-edge transition in AIN is allowed only for the $\mathbf{E} \| \mathbf{c}$ polarization and almost forbidden for the $\mathbf{E} \perp \mathbf{c}$ polarization. Therefore, spectral peak position, line width, and observed 


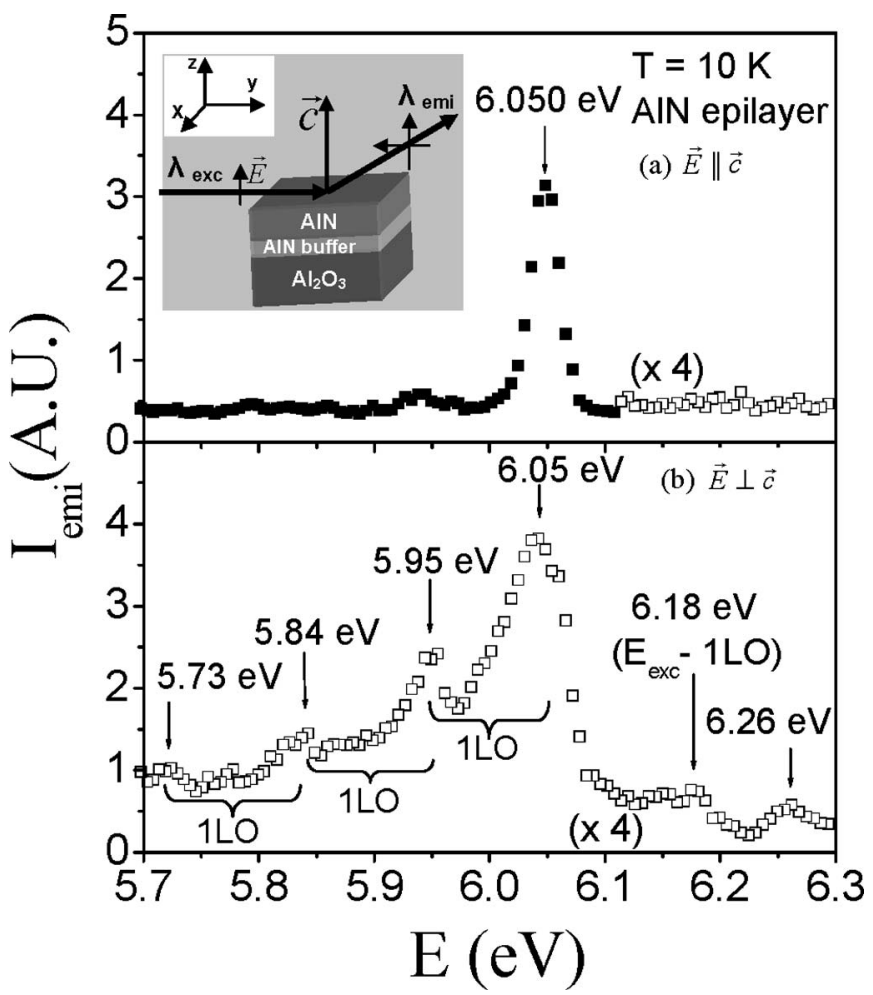

FIG. 1. Low temperature (10 K) PL spectra of an AlN epilayer measured under different polarization configurations with (a) $\mathbf{E} \| \mathbf{c}$ and (b) $\mathbf{E} \perp \mathbf{c}$. Schematic diagram of the measurement setup is shown in the inset of (a).

relative intensities of two polarization resolved measurements suggest that the $6.05 \mathrm{eV}$ peak is due to the recombination of $A$-free excitons ( $A$-FX). Using the binding energy of $A$-FX in AlN of $80 \mathrm{meV}$, the band gap of AlN is $(6.05$ $+0.08 \mathrm{eV})=6.13 \mathrm{eV}$ at $10 \mathrm{~K}$, which is consistent with the previously reported values. ${ }^{13-15}$ A slight variation in the FX emission peak position from previous reports is due to different magnitudes of stress in different samples caused by variations in growth conditions, layer thickness, etc. ${ }^{16}$

For the $\mathbf{E} \perp \mathbf{c}$ orientation shown in Fig. 1(b), in addition to the $6.05 \mathrm{eV}$ peak, there is another emission line at a higher energy position near $6.26 \mathrm{eV}$, which is absent in the $\mathbf{E} \| \mathbf{c}$ measurement configuration. In Fig. 2, we replot the same emission spectrum of Fig. 1(b) in a narrower spectral range from 6.20 to $6.35 \mathrm{eV}$ with higher resolution. Two peaks at

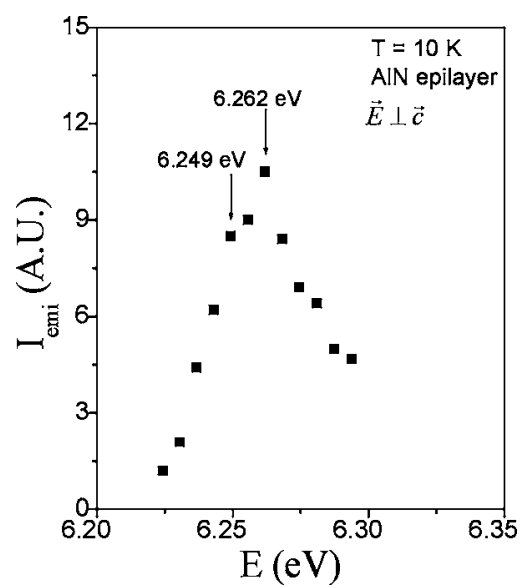

FIG. 2. Low temperature PL spectra of an AlN epilayer for $\mathbf{E} \perp \mathbf{c}$ polarization in a narrow spectral range. rameters are related by
Downloaded 12 Jul 2010 to 129.118 .86 .45 . Redistribution subject to AIP license or copyright; servations possible. tively.
6.249 and $6.262 \mathrm{eV}$ are clearly resolved only for $\mathbf{E} \perp \mathbf{c}$ polarization. These two peaks are separated by $13 \mathrm{meV}$. Previous theoretical calculations ${ }^{1,2}$ have predicted that the energy separation between the $B$ and $C$ valence bands is $13 \mathrm{meV}$ and that between the $A$ and $B$ valence bands is $\sim 211 \mathrm{meV}$. The observed peak at $6.249 \mathrm{eV}$ in Fig. 1(b) is about $199 \mathrm{meV}$ higher than the $A$-exciton peak at $6.050 \mathrm{eV}$. Therefore, based on the calculations, observed spectral peak positions and relative emission intensities in two different polarization orientations, we assign the emission peaks at 6.249 and $6.262 \mathrm{eV}$ to the $B$ and $C$ exciton recombination, respectively. These two emission bands have not been previously observed by direct PL measurements. We believe that improved crystalline quality of AlN epilayers makes their ob-

A previous optical reflectance measurement revealed a spectral peak position at about $6.029 \mathrm{eV}$ for the $A$-FX transition in AlN bulk crystals, ${ }^{6}$ which matches perfectly with that in AIN homo-epilayers obtained by PL measurements ${ }^{16}$ and represents the $A$-FX transition peak in stress-free AlN. It was not possible to fully resolve the peak positions of $B$ - and $C$-exciton transitions by the optical reflectance measurement but careful fitting suggested positions at 6.243 and $6.268 \mathrm{eV}$, respectively. ${ }^{6}$ The $A$ - and $B$-exciton emission peaks observed here (in a $4 \mu \mathrm{m}$ thick $\mathrm{AlN} / \mathrm{Al}_{2} \mathrm{O}_{3}$ epilayer) are blueshifted by 21 and $6 \mathrm{meV}$, respectively, with respect to the stress-free AlN due to a compressive strain, while a blueshift for the $C$-exciton transition is absent based on this optical reflectance measurement. ${ }^{6}$ A separate optical reflectance/ transmission measurement on AlN with various crystal orientations revealed that the $A, B$, and $C$ excitons have transition energies of $6.025,6.243$, and $6.257 \mathrm{eV}$ in stressfree $\mathrm{AlN},{ }^{5}$ which implies that the $A, B$, and $C$ exciton emission lines observed here (in a $4 \mu \mathrm{m}$ thick $\mathrm{AlN} / \mathrm{Al}_{2} \mathrm{O}_{3}$ epilayer) are blueshifted by 25,6 , and $5 \mathrm{meV}$, respectively, with respect to the stress-free AlN due to a compressive strain. The results seem to suggest that the effects of strain on $B$ and $C$ valence bands are less pronounced than that on the $A$ valence band. However, the effects of strain on $B$ - and $C$-valence bands are still ambiguous at this point. We are unable to deduce the binding energies of the $B$ and $C$ excitons in AlN based on the present experimental data. However, excitons associated with the $A, B$, and $C$ valence bands have almost the same binding energies in GaN. ${ }^{17}$ Therefore, we assume that the $B$ and $C$ excitons in AlN have the same binding energy as that of the $A$ exciton. This gives the band gap of AlN to be $(6.249+0.080)=6.329 \mathrm{eV}$ and $(6.262$ $+0.080)=6.342 \mathrm{eV}$ for the $B$ and $C$ valence bands, respec-

Based on the results shown in Fig. 1, we have constructed in Fig. 3 the energy band structure of AlN near the $\Gamma$ point. The corresponding optical transitions are illustrated by downward arrows. Polarization properties of emitted light are different depending on which of the valence bands are involved in the recombination. Recombination of the $B$ and $C$ excitons are allowed in the $\mathbf{E} \perp \mathbf{c}$ polarization configuration and forbidden in the $\mathbf{E} \| \mathbf{c}$ polarization configuration, which is in contrast to the $A$ exciton recombination that is allowed mainly in the $\mathbf{E} \| \mathbf{c}$ polarization configuration and almost prohibited in the $\mathbf{E} \perp \mathbf{c}$ polarization configuration. According to Hopfield's quasicubic model for WZ crystals, ${ }^{18}$ excitonic emission energies and valence band splitting pa-

Hedistribution subject to AlP license or copyright; see http://apl.aip.org/apl/copyright.jsp 


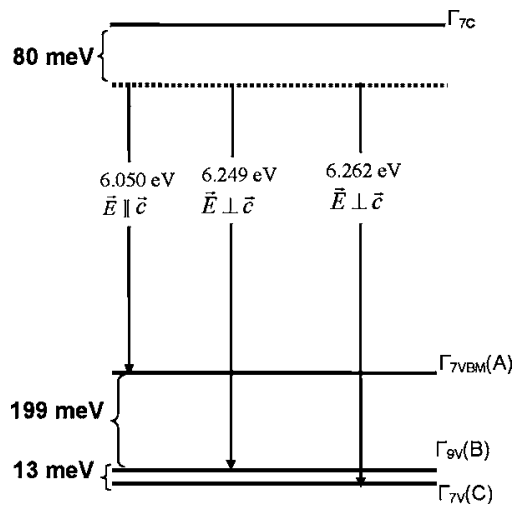

FIG. 3. Energy level diagram of AlN near the $\Gamma$ point constructed from the low temperature PL measurement results.

$$
E_{\mathrm{A} / \mathrm{C}}=E_{B}+\frac{\Delta_{\mathrm{SO}}+\Delta_{\mathrm{CR}}}{2} \mp \sqrt{\left(\frac{\Delta_{\mathrm{SO}}+\Delta_{\mathrm{CR}}}{2}\right)^{2}-\frac{2}{3} \Delta_{\mathrm{SO}} \Delta_{\mathrm{CR}}},
$$

where $E_{A / C}\left(E_{B}\right)$ are the emission energies from the band-toband transitions related to $A / C(B)$ valence bands, respectively. Using our experimentally observed values $E_{A}$ $=6.050 \mathrm{eV}, E_{B}=6.249 \mathrm{eV}$, and $E_{C}=6.262 \mathrm{eV}$, we deduce $\Delta_{\mathrm{CR}}=-206 \mathrm{meV}$ and $\Delta_{\mathrm{SO}}=20 \mathrm{meV}$ for AlN epilayer grown on sapphire substrate.

Values of $\Delta E_{A B}, \Delta E_{B C}, \Delta_{\mathrm{CR}}$, and $\Delta_{\mathrm{SO}}$ from previous theoretical calculations and experimental observations are summarized in Table I along with the respective methods of experiments. Bearing in mind that different amounts of strains involved for AlN epilayers grown on different substrates with varying thicknesses, our data agree well with majority of the reported experimental and calculated values. The intensities of these two emission lines are relatively weak, which seems reasonable. Typically, transitions involving higher bands such as the $B$ and $C$ valence bands are very

TABLE I. Theoretically calculated and experimentally observed values of spin-orbit splitting parameter $\left(\Delta_{\mathrm{SO}}\right)$, crystal-field splitting parameter $\left(\Delta_{\mathrm{CR}}\right)$, energy spacing between the $A$ and $B$ valence bands $\left(E_{A B}\right)$, and the $B$ and $C$ valence bands $\left(\Delta E_{B C}\right)$ in WZ AlN.

\begin{tabular}{|c|c|c|c|c|}
\hline $\begin{array}{c}\Delta_{\mathrm{SO}} \\
(\mathrm{meV})\end{array}$ & $\begin{array}{c}\Delta_{\mathrm{CR}} \\
(\mathrm{meV})\end{array}$ & $\begin{array}{l}\Delta E_{A B} \\
(\mathrm{meV})\end{array}$ & $\begin{array}{c}\Delta E_{B C} \\
(\mathrm{meV})\end{array}$ & Notes \\
\hline & -219 & 213 & 13 & Calc. $^{a}$ \\
\hline 19 & -217 & 211 & 13 & Calc. ${ }^{\mathrm{b}}$ \\
\hline \multirow[t]{3}{*}{19} & -224 & 218 & 19 & Calc. $^{c}$ \\
\hline & -237 & \multicolumn{2}{|c|}{$\begin{array}{c}E_{B}, E_{C}=6.22 \mathrm{eV} \text { not resolved } \\
E_{\mathrm{A}}=6.024 \mathrm{eV}\end{array}$} & $\begin{array}{c}\text { OR, CL, PL } \\
\text { AlN:Si/SiC }\end{array}$ \\
\hline & -230 & \multicolumn{2}{|c|}{$E_{B}, E_{C}=6.25 \mathrm{eV}$ not resolved } & OR, $m$-face bulk AlN ${ }^{\mathrm{e}}$ \\
\hline 36 & -225 & 234 & 25 & OR, bulk AlN ${ }^{\mathrm{e}}$ \\
\hline 20 & -206 & 199 & 13 & This work, $\mathrm{AlN} / \mathrm{Al}_{2} \mathrm{O}_{3}$ \\
\hline
\end{tabular}

$\overline{\text { Calc.: calculation, OR: optical reflectivity, CL: cathodoluminescence } E_{A}, E_{B}}$, $E_{C}$ : emission energies corresponding to the $A, B$ and $C$ valence bands.

$\begin{array}{ll}{ }^{\mathrm{a}} \text { Reference } 1 . & { }^{\mathrm{d}} \text { Reference } 4 . \\ { }^{\mathrm{b}} \text { Reference } 2 . & { }^{\mathrm{e}} \text { Reference } 5 . \\ { }^{\mathrm{c}} \text { Reference } 3 . & { }^{\mathrm{f}} \text { Reference } 6 .\end{array}$

difficult to observe due to a much lower population of free holes, in accordance with the statistical distribution. ${ }^{19}$ The free hole concentration is much higher in the $A$ valence band. However, in the case here, the transitions from the $B$ and $C$ valence bands are enhanced due to the unique polarization properties of these bands in AlN. Nevertheless, other complementary measurements such as high resolution optical absorption measurements may be needed in order to further confirm the energetic positions of $B$ and $C$ exciton transitions.

In summary, we have investigated the valence band structure of AlN near the $\Gamma$ point by polarization resolved deep UV PL measurements. Three different emission lines have been observed. As expected, the $A$ valence band related excitonic emission at $6.050 \mathrm{eV}$ with $\mathbf{E} \| \mathbf{c}$ polarization dominates the spectrum. The observed $B$ and $C$ valence bands related excitonic emissions at higher energy positions of 6.249 and $6.262 \mathrm{eV}$ are polarized in the $\mathbf{E} \perp \mathbf{c}$ direction. From these results, the crystal-field and spin-orbit splitting parameters are deduced, which agree well with previous reported results. We believe that the present results provide a more coherent picture for the valence band structure of the WZ AlN.

This work is supported by a grant from DOE (DEFGOR-96ER 45604).

${ }^{1}$ J. Li, K. B. Nam, M. L. Nakarmi, J. Y. Lin, and H. X. Jiang, P. Carrier, and S.-H. Wei, Appl. Phys. Lett. 83, 5163 (2003).

${ }^{2}$ S.-H. Wei and A. Zunger, Appl. Phys. Lett. 69, 2719 (1996).

${ }^{3}$ P. Carrier and S.-H. Wei, J. Appl. Phys. 97, 033707 (2005).

${ }^{4}$ G. I. M. Prinz, A. Ladenburger, M. Schirra, M. Feneberg, K. Thonke, R. Sauer, Y. Taniyasu, M. Kasu, and T. Makimoto, J. Appl. Phys. 101, 023511 (2007).

${ }^{5}$ L. Chen, B. J. Skromme, R. F. Dalmau, R. Schlesser, Z. Sitar, C. Chen, W. Sun, J. Yang, M. A. Khan, M. L. Nakarmi, J. Y. Lin, and H. X. Jiang, Appl. Phys. Lett. 85, 4334 (2004).

${ }^{6}$ E. Silveria, J. A. Freitas, Jr., O. J. Glembocki, G. A. Slack, and L. J. Schowalter, Phys. Rev. B 71, 041201 (2005).

${ }^{7}$ M. Suzuki, T. Uenoyama, and A. Yanase, Phys. Rev. B 52, 8132 (1995).

${ }^{8}$ C. Y. Yeh, Z. W. Lu, S. Froyen, and A. Zunger, Phys. Rev. B 46, 10086 (1992).

${ }^{9}$ K. B. Nam, J. Li, M. L. Nakarmi, J. Y. Lin, and H. X. Jiang, Appl. Phys. Lett. 84, 5264 (2004)

${ }^{10}$ B. N. Pantha, R. Dahal, M. L. Nakarmi, N. Nepal, J. Li, J. Y. Lin, H. X. Jiang, Q. S. Paduano, and D. Weyburne, Appl. Phys. Lett. 90, 241101 (2007).

${ }^{11}$ J. Li, Z. Y. Fan, R. Dahal, M. L. Nakarmi, J. Y. Lin, and H. X. Jiang, Appl. Phys. Lett. 89, 213510 (2006).

${ }^{12} \mathrm{See}$ (http://www.phys.ksu.edu/area/GaNgroup).

${ }^{13}$ J. Li, K. B. Nam, M. L. Nakarmi, J. Y. Lin, and H. X. Jiang, Appl. Phys. Lett. 81, 3365 (2002).

${ }^{14}$ K. B. Nam, M. L. Nakarmi, J. Li, J. Y. Lin, and H. X. Jiang, Appl. Phys. Lett. 83, 878 (2003).

${ }^{15}$ N. Nepal, M. L. Nakarmi, H. U. Jang, J. Y. Lin, and H. X. Jiang, Appl. Phys. Lett. 89, 192111 (2006).

${ }^{16}$ B. N. Pantha, N. Nepal, T. M. Al Tahtamouni, M. L. Nakarmi, J. Li, J. Y. Lin, and H. X. Jiang, Appl. Phys. Lett. 91, 121117 (2007).

${ }^{17}$ A. Wysmolek, M. Potemski, R. Stepniewski, J. Lusakowski, K. Pakula, J. M. Baranowski, G. Martinez, P. Wyder, I. Grzegory, and S. Porowski, Phys. Status Solidi A 216, 11 (1999).

${ }^{18}$ J. J. Hopfield, J. Phys. Chem. Solids 15, 97 (1960).

${ }^{19}$ B. Šantić, Semicond. Sci. Technol. 18, 219 (2003). 